\title{
Diagnostic Value of Diffusion Magnetic Resonance Imaging in Detecting Malignant Axillary Lymph Nodes in Breast Cancer Patients
}

\author{
- Betul Duran, (1) Burcin Agridag Ucpinar \\ University of Health Sciences Turkey, Sisli Etfal Training and Research Hospital, Clinic of Radiology, Istanbul, Turkey
}

\section{Abstract}

\begin{abstract}
Aim: The prognostic value of apparent diffusion coefficient (ADC) measurement to detect axillary malignant lymph nodes in breast cancer is not clear. We aimed to investigate the prognostic importance of diffusion weighted imaging (DWI) in the differentiation of metastatic and non-metastatic lymph nodes.

Methods: Magnetic resonance imagings (MRIs) of 102 breast cancer patients were reviewed from the hospital PACS and automation systems between July 2018 and March 2021. Cortical thickness, short, long-axis diameters, long/short axis ratios and ADC measurements were done for ipsilateral and contralateral axillary lymph nodes. Univariate and multivariate regression analyses were performed.

Results: Pathology revealed that 64 patients had ipsilateral metastatic lymph nodes while 38 patients did not. When grouped into metastatic and non-metastatic groups, breast tumor size, mean diameter of the short axis, long axis and cortical thickness were significantly higher in the metastatic group. Ipsilateral metastatic lymph nodes' mean ADC values were significantly lower than nonmetastatic ones with a calculated value of $0.972 \times 10^{-3}$. The ipsilateral ADC value of the lymph node and loss of fatty hilum were the two significant factors to predict metastatic lymph nodes in multivariate analysis.
\end{abstract}

Conclusion: DWI is a valuable noninvasive tool in the diagnosis of metastatic axillary lymph nodes.

Keywords: Breast neoplasm, axilla, lymph nodes

\section{Introduction}

Breast cancer is the most common type of malignancy and the second most common cause of death among women in Turkey as well as in the world $(1,2)$. The presence of lymph node metastasis is the most important prognostic factor for long-term and disease-free survival in patients $(3,4)$. Axillary imaging of newly diagnosed breast cancer patients is currently done by either ultrasonography (US) or magnetic resonance imaging (MRI). MRI has advantages over US as being non-operator dependent, enabling it to evaluate even the deeply located lymph nodes and compare with contralateral axilla simultaneously. Conventional MRI features suspicious for metastatic axillary nodes are reported as cortical thickening, loss of fatty hilum, round shape and heterogeneous contrast enhancement after Gadolinium injection (5). Diffusionweighted imaging (DWI) is an advanced functional MRI method that apparents the free motion of the water molecules in tissues (6). Apparent diffusion coefficient (ADC) can quantify the aforementioned motion of the water molecules and provide information about the microscopic cellular changes like cellular membrane integrity or cell proliferation $(7,8)$.

So far, the prognostic value of ADC measurement of the lymph nodes in various malignities of head and neck, uterus and cervix have been shown (9). However, it is not clear whether it contributes to detecting axillary malignant lymph nodes in breast cancer. In the review of De Cataldo et al. (10) they concluded that DWI could be used in patients with low-intermediate risk of lymph node involvement. In another study of Liu et al. (11) they didn't find significant differences regarding ADC values of metastatic and nonmetastatic axillary lymph nodes in T1 and T2 stage breast cancer patients. In this study,

Address for Correspondence: Betul Duran, University of Health Sciences Turkey, Sisli Etfal

Training and Research Hospital, Clinic of Radiology, Istanbul, Turkey

E-mail: betulduran_@outlook.com ORCID: orcid.org/0000-0001-8598-6814

Received: 19.06.2021 Accepted: 15.10.2021

Copyright 2021 by The Medical Bulletin of istanbul Haseki Training and Research Hospital The Medical Bulletin of Haseki published by Galenos Yayınevi. 
we aimed to investigate the prognostic importance of DWI in the differentiation of ipsilateral metastatic and non-metastatic lymph nodes and determine the optimal combination of the MRI features to detect nodal status in breast cancer patients.

\section{Methods}

\section{Study Design}

This study was conducted in accordance with the 1964 Helsinki declaration and approval of the Ethics Committee of the hospital was obtained. Informed consent was waived. The cohort of the study was 114 newly diagnosed breast cancer patients at our radiology clinic who underwent preoperative breast MRI and US-guided ipsilateral axillary lymph node biopsy between July 2018 and March 2021. Patients who underwent axillary lymph node biopsy before breast MRI $(n=4)$, patients whose DWI had artefacts which impede appropriate interpretation $(n=2)$ and patients whose histopathological data couldn't be recollected $(n=6)$ were excluded from this study. After inclusion and exclusion criteria were applied, 102 patients with 102 ipsilateral lymph nodes were enrolled in this study. All patients underwent tru-cut biopsy from breast masses and sentinel lymph node biopsy (SLNB) or axillary dissection from the ipsilateral axilla.

\section{Breast MRI Protocol}

All breast MRIs were acquired using 1.5 Tesla Siemens scanner (Avanto, Erlengen Germany) with patients positioned prone in an 8-channel breast array coil. The conventional MRI protocol was applied as T1 weighted fast spin echo axial sequence $(T R=650, T E=112$, Matrix $448 \times 224, \mathrm{FOV}=320 \times 320 \mathrm{~mm}, \mathrm{NEX}=1$, Thickness $=$ $3.0 \mathrm{~mm}$ ) and pre- and post-contrast T1 weighted threedimensional fat-suppressed axial sequence $(T R=485$, TE $=10$, Matrix $350 \times 350$, FOV $=320 \times 320 \mathrm{~mm}, \mathrm{NEX}=$ $1, F A=10.0$, Thickness 3.00). Images were taken before contrast administration and five times after contrast injection with 80s intervals. Gadopentetate dimeglumine contrast medium was injected with a dose of $0.1 \mathrm{mmol}$ $\mathrm{kg}-1$. Diffusion-weighted images [TR/TE $=1000 / 83$, NEX $=2$ and Thickness $=2 \mathrm{~mm}, \mathrm{FOV}=320 \mathrm{~mm}$, Matrix 180x238] were obtained before contrast and ADC maps were attained. To interpret DWI, b0 and b1000 sn/mm² were used.

\section{Evaluation of Conventional and Diffusion Weighted MRIs}

All of the MRI images were analyzed and reported by consensus readings of two radiologists with 8 years and 4 years of experience in breast radiology. The interpreters were blinded to histopathological results. In conventional MRI sequences, presence or loss of fatty hilum, cortical thickness, long-axis diameter, short-axis diameter, longaxis to short-axis ratios were evaluated. While loss of fatty hilum was evaluated in precontrast T1 and T2 weighted images, other features were evaluated in precontrast $\mathrm{T} 2$ weighted images. The index lymph node was selected in consensus if there was no suspicious lymph node in axilla. While calculating ADC values, freely selected minimum size regions of interests (ROIs) were used and located to the cortex of each lymph node. Three measurements from the same and most suspicious lymph nodes were done and the minimum values were noted. Attention was given while placing ROIs not to include fatty hilar or adjacent soft tissues (Figure 1,2). The same calculation method was used to compare the contralateral axillary lymph nodes in normal morphology.

\section{Histopathologic Evaluation}

Histopathology results were obtained by fine needle aspiration biopsy of the most suspicious lymph nodes, SLNB or axillary curettage. In patients with suspicious metastatic lymph nodes in MRI images, care was taken to biopsy this lymph node. In patients with normal morphological lymph nodes, preoperative biopsy was not planned and SLNB was used as the histopathologic indicator.

\section{Statistical Analysis}

For statistical analysis, SPSS 15.0 for Windows program was used. Continuous data (short axis length, long axis length, long axis-short axis ratio, maximal cortical thickness, and ADC value) are given as mean, standard deviation, minimum, maximum, median, interquartile range. Categorical variables (sex, loss of fatty hilum) are

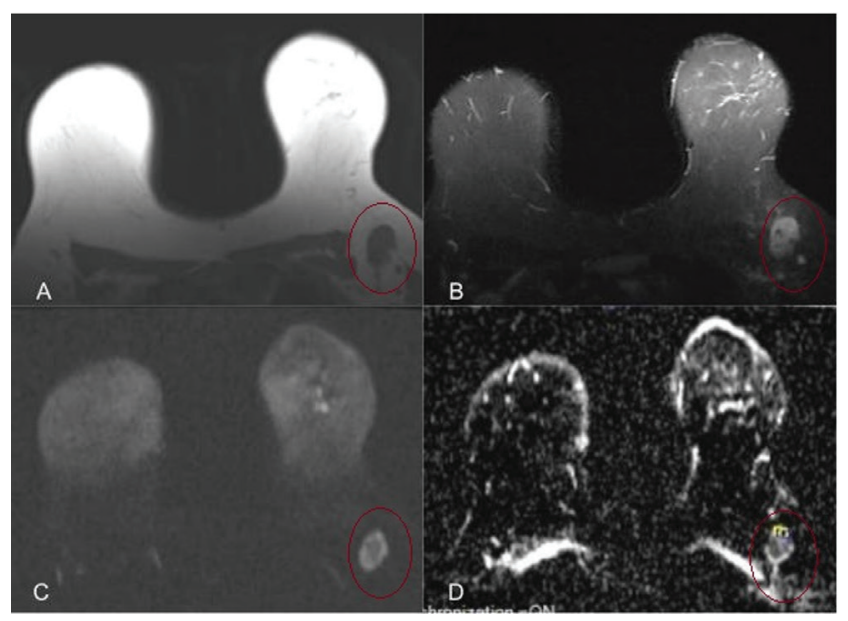

Figure 1. Sixty-eight year-old female patient diagnosed with invasive ductal carcinoma of the left breast with axillary metastasis. A) T1- weighted, B) T2- weighted, C) Diffusion -weighted imagings and D) ADC map. An enlarged metastatic lymph node is seen in the left axilla which shows prominent diffusion restriction ADC: Apparent diffusion coefficient 


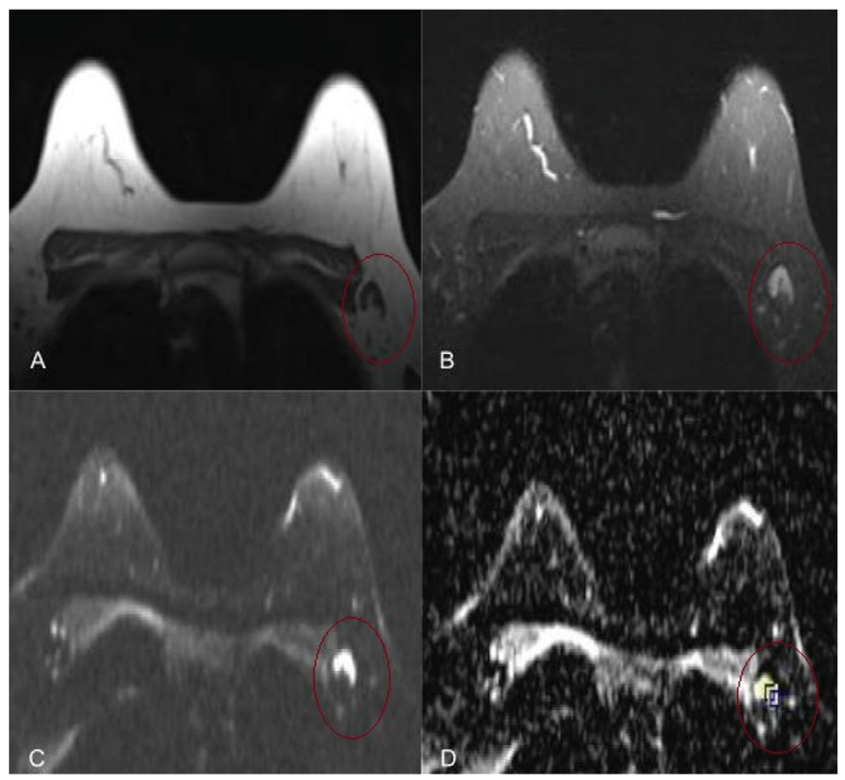

Figure 2. Forty-three year-old female patient diagnosed with tubular carcinoma of the left breast. There is a lymph node in the left axilla. A) T1-weighted and B) T2 weighted imaging) which is proven as reactive pathologically. No diffusion restriction is seen in C)DWI and D) ADC map

ADC: Apparent diffusion coefficient, DWI: Diffusion weighted imaging

\section{Ipsilateral lymph nodes}

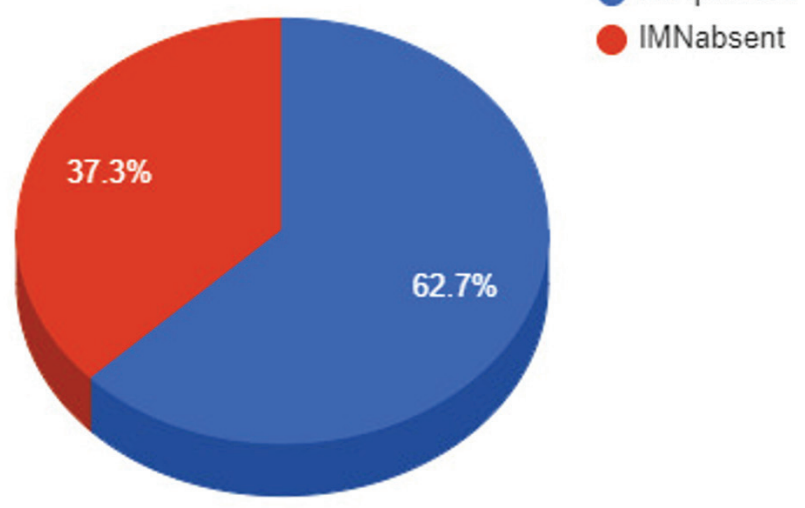

Figure 3. Schematic representation of ipsilateral metastatic nodes present (IMNpresent) and absent (IMNabsent) patients

given as numbers and percentage. Independent two-group comparisons were achieved with the Student's t-test when the numerical variables met the normal distribution condition, and the Mann-Whitney U-test was used when the condition was not met. Relationships between numerical variables were performed using Spearman correlation analysis since parametric test conditions were not met. To compare independent samples, the Wilcoxon test was used. To obtain cut-off values and compare the diagnostic performance of variables, receiver operating characteristics (ROC) was used. The optimal cut-off value was determined according to the highest Youden index $(J)$, and the sensitivity, specificity, positive predictive value (PPV), and negative predictive value (NPV) were calculated for each variable. Determinative factors were examined by logistic regression analysis. Statistical significance level of alpha was accepted as $p<0.05$.

\section{Results}

The mean patient age was 52.0 \pm 11.9 (range between 32 and 85). The most common pathological subtype was invasive ductal carcinoma ( $n=73,71.57 \%)$ followed by invasive lobular carcinoma ( $n=9,8.82 \%)$. FNAB or surgical final pathology revealed that 64 patients had ipsilateral the metastatic lymph nodes while 38 patients did not (Figure 1). Loss of fatty hilum was present in 38 ipsilateral lymph nodes $(37.3 \%)$, whereas $64(62.7 \%)$ of them had normal hilar structure.

When grouped into metastatic and non-metastatic ipsilateral lymph nodes, breast tumor size $(p=0.004)$, mean diameter of the short axis $(p=0.001)$, long axis $(p=0.003)$ and cortical thickness $(p<0.001)$ were significantly higher in metastatic group. There were no statistical differences between the age of the patients and long/short axis ratios between the two groups (Table 1)

Ipsilateral metastatic lymph nodes' mean ADC values were significantly lower than ipsilateral non-metastatic lymph nodes $(p<0,001)$, as expected. The mean ADC value of contralateral lymph nodes was significantly higher in the ipsilateral metastatic lymph node group $(p<0,009)$. There was a statistically significant difference in the metastatic lymph node group between ipsilateral and contralateral $A D C$ values $(p<0.001)$ whereas not in the non-metastatic group $(p<0.08)$ (Table 2$)$.

ROC curve analysis was performed and area under curves (AUC) were calculated for ADC values and each conventional MRI feature to predict metastatic ipsilateral lymph nodes. The highest Jouden indices and AUC values were calculated in ipsilateral ADC value and cortical thickness parameters (Figure 3). The cut of the value of ADC was found $0.972 \times 10-3$ with $84.4 \%$ sensitivity and $86.8 \%$ specificity, 91.5\% PPV, 76.7\% NPV (J: 71.22, AUC: 0.929$)$. The cut off value of cortical thickness was found 5.5 millimeters with $76.56 \%$ sensitivity and $81.58 \%$ specificity, 87,5\% PPV, 67.39\% NPV (J: 57.77 AUC: 0.828). The lowest AUC and sensitivity, specificity, PPV and NPV were observed in the long axis-short axis ratio (Table 3).

Fatty hilum loss was the only descriptive parameter in our study. The number of fatty hilum loss in lymph metastatic group lymph nodes was significantly higher 
than ones in the non-metastatic group. Even the sensitivity was relatively low (54.7\%), the specificity $(92.1 \%)$ and PPV (92.1\%) were significantly high.

Multiple regression analysis was performed to investigate the effect of calculated cut-off value on other conventional MRI features. The ipsilateral ADC value of the lymph node and loss of fatty hilum were the two significant factors to predict metastatic lymph nodes (Table 4).

\section{Discussion}

Axillary lymph node status is the most important prognostic factor in newly diagnosed breast cancer patients
(9). Detecting axillary metastasis in breast cancer patients is crucial as it has a primary role in staging and optimal treatment decision. The diagnostic values of physical examination, ultrasound-guided biopsy, mammography and ultrasonography were evaluated in the prediction of axillary involvement in breast cancer patients and the results were not satisfactory (12). Nowadays; SLNB is the gold standard technique with high sensitivity of $91.2 \%$ and high specificity of almost $100 \%$ for the detection of metastatic lymph nodes (13). However; it is an invasive procedure with possible complications.

At this point, as being a noninvasive alternative, conventional MRI with dynamic contrast enhancement

Table 1. Age, tumor size of the patients and short diameter, long diameter, long to short diameter ratio and cortical thickness parameters in ipsilateral metastatic and non-metastatic lymph nodes

\begin{tabular}{|c|c|c|c|c|c|}
\hline & \multicolumn{4}{|c|}{ Lymph node status } & \multirow[b]{3}{*}{$p^{*}$} \\
\hline & \multicolumn{2}{|l|}{ Metastatic } & \multicolumn{2}{|c|}{ Non-metastatic } & \\
\hline & Mean \pm SD & Median (IQR) & Mean \pm SD & Median (IQR) & \\
\hline Age & $51.6 \pm 11.3$ & $50(43.25-59)$ & $52.6 \pm 13.1$ & $50(42.75-61)$ & 0.928 \\
\hline Tumor size & $34.0 \pm 19.5$ & $31(20-40)$ & $24.8 \pm 13.9$ & $23.5(15-29.5)$ & 0.004 \\
\hline Short axis diameter & $12.86 \pm 5.64$ & $11(9-17)$ & $9.29 \pm 4.37$ & $8.5(6-11)$ & 0.001 \\
\hline Long axis diameter & $21.39 \pm 9.12$ & $19(14.25-27.5)$ & $16.16 \pm 6.42$ & $16(12-18)$ & 0.003 \\
\hline Long/short axis diameter & $1.73 \pm 0.49$ & $1.6(1.43-1.87)$ & $1.79 \pm 0.37$ & $1.75(1.55-2.02)$ & 0.156 \\
\hline Cortical thickness & $10.59 \pm 6.06$ & $9(6-14)$ & $4.97 \pm 2.54$ & $5(4-5)$ & $<0.001$ \\
\hline
\end{tabular}

Table 2. Mean and Median ADC values of ipsilateral metastatic and non-metastatic lymph nodes and the contralateral lymph nodes of all patients

\begin{tabular}{|c|c|c|c|c|c|}
\hline & \multicolumn{4}{|c|}{ Lymph node status } & \multirow[b]{3}{*}{$\mathbf{p}^{*}$} \\
\hline & \multicolumn{2}{|l|}{ Metastatic } & \multicolumn{2}{|c|}{ Non-metastatic } & \\
\hline & Mean \pm SD & Median (IQR) & Mean \pm SD & Median (IQR) & \\
\hline Ipsilateral ADC $\left(\times 10^{-3}\right)$ & $0.77 \pm 0.19$ & $0.76(0.62-0.90)$ & $1.17 \pm 0.20$ & $1.15(1.07-1.31)$ & $<0.001$ \\
\hline Contralateral ADC $\left(\times 10^{-3}\right)$ & $1.27 \pm 0.21$ & $1.27(1.09-1.40)$ & $1.15 \pm 0.18$ & $1.12(1.04-1.27)$ & 0.009 \\
\hline$p^{* *}$ & \multicolumn{2}{|l|}{$<0.001$} & \multicolumn{2}{|l|}{$0.080^{a}$} & \\
\hline
\end{tabular}

${ }^{*}$ Ipsilateral $A D C p<0.001$ by 2-sample t-test, Contralateral ADC $p=0.009$ by Mann-Whitney $U$ test, **by Wilcoxon test, ${ }^{a}$ There was no significant difference only when ipsilateral and contralateral ADC values were compared in non-metastatic group, SD: Standard deviation, IQR: Interquartile range, ADC: Apparent diffusion coefficient

\begin{tabular}{|c|c|c|c|c|c|c|c|c|c|c|}
\hline Test result variable (s) & AUC & $95 \% \mathrm{C}$ & & p & $\begin{array}{l}\text { Cut-off } \\
\text { value }\end{array}$ & $\begin{array}{l}\text { Youden } \\
\text { index }\end{array}$ & $\mathrm{Se}(\%)$ & $\mathrm{Sp}(\%)$ & PPV (\%) & NPV (\%) \\
\hline Primary tumor size & 0.673 & 0.567 & 0.779 & 0.004 & 24.5 & 16.12 & 63.49 & 52.63 & 68.97 & 46.51 \\
\hline Cortical thickness & 0.828 & 0.746 & 0.910 & $<0.001$ & 5.5 & 57.77 & 76.56 & 81.58 & 87.5 & 67.39 \\
\hline Short axis diameter & 0.701 & 0.599 & 0.804 & 0.001 & 9.5 & 30.37 & 70.31 & 60.53 & 75.00 & 54.76 \\
\hline Long axis diameter & 0.670 & 0.565 & 0.775 & 0.004 & 16.5 & 25.61 & 65.63 & 60.53 & 73.68 & 51.11 \\
\hline $\begin{array}{l}\text { Long axis/short axis } \\
\text { diameter }\end{array}$ & 0.421 & 0.306 & 0.535 & 0.183 & 1.696 & 11.02 & 53.13 & 57.89 & 68.00 & 42.31 \\
\hline $\begin{array}{l}\text { Ipsilateral } \\
\text { ADC }\left(\times 10^{-3}\right)\end{array}$ & 0.929 & 0.018 & 0.124 & $<0.001$ & 0.972 & 71.22 & 84.38 & 86.84 & 91.53 & 76.74 \\
\hline
\end{tabular}


stands out to evaluate the nodal status. Preoperative conventional MRI gives the opportunity to staging,

\begin{tabular}{|c|c|c|c|c|}
\hline \multirow[b]{3}{*}{$\begin{array}{l}\text { Ipsilateral ADC }\left(\times 10^{-3}\right) \\
<0.972\end{array}$} & \multirow{3}{*}{$\begin{array}{l}\mathbf{p} \\
<0.001\end{array}$} & \multirow{3}{*}{$\begin{array}{l}\text { OR } \\
77.896\end{array}$} & \multirow{2}{*}{\multicolumn{2}{|c|}{ \%95 Cl (min-max) }} \\
\hline & & & & \\
\hline & & & 11.838 & 512.588 \\
\hline Loss of fatty hilum & 0.006 & 65.541 & 3.260 & 1317.507 \\
\hline Cortical thickness & 0.736 & 0.920 & 0.568 & 1.492 \\
\hline Short axis diameter & 0.542 & 0.736 & 0.275 & 1.973 \\
\hline Long axis diameter & 0.289 & 1.409 & 0.748 & 2.653 \\
\hline $\begin{array}{l}\text { Long/short axis } \\
\text { diameter }\end{array}$ & 0.304 & 0.053 & 0.000 & 14.281 \\
\hline
\end{tabular}

detecting and measurement of axillary lymph nodes and evaluation of morphological features like cortical thickness, shape, fatty hilum obliteration, and contrast enhancement $(14,15)$. In recent years; DWI has been added to routine breast MRI protocols to increase diagnostic performance. DWI is widely used in diagnosis and treatment response in breast cancer (16-18). The value of DWI in axillary lymph node evaluation is still controversial.

This study showed the diagnostic performance of DWI and conventional MRI characteristics of ipsilateral lymph nodes to predict malignancy in breast cancer patients. Regarding conventional MRI characteristics; long axis, short axis and cortical thickness of the malignant lymph nodes were significantly higher in the metastatic group. The ratio of long axis to short axis sizes did not differ between groups. In previous studies, no significant correlation was found between lymph node diameter and the presence of metastasis (19-22). However, Atallah et al. (23) and Yoshimura et al. (24) found that metastatic lymph nodes had larger long-axis size with a threshold of 12 and 10, respectively. In our study, we calculated the short and long-axis diameters as $9.5 \mathrm{~mm}$ and $11.5 \mathrm{~mm}$. In another study, these values were found 9.3 and $11.3 \mathrm{~mm}$ which are very similar to our results (9). We found that the cortical thickness was the best diagnostic parameter among conventional MRI features. Kim et al. (9) and Scaranelo et al. (25) also found the maximal cortical thickness as the most discriminative parameter among conventional MRI features.

As being the only descriptive parameter; fatty hilum loss was observed in 3 (7.89\%) cases among the nonmetastatic group and 35 (54.69\%) cases among the metastatic group. Fatty hilum loss showed the highest specificity $(92.1 \%)$ that loss of fatty hilum is the best parameter to exclude the metastatic lymph nodes. This result was concordant with the literature $(9,24,26)$.
In this study, the cut-off $A D C$ value of the ipsilateral lymph node $<0,972 \times 10^{-3}$ showed highest sensitivity $(84.3 \%)$, specificity (86.4\%), PPV (91.53\%) and NPV (76.74\%) to predict the nodal status. In terms of ADC value measurement, our results were concordant with the literature (27-29). Guvenc et al. (27) found the cut-off value of $A D C$ value as $0.985 \times 10^{-3} \mathrm{~mm}^{2} / \mathrm{sec}$ with a sensitivity of $83 \%$, specificity of $98 \%$, PPV of $95 \%$, and NPV of $93 \%$. They calculated the AUC for ipsilateral lymph nodes as 0.96. Kim et al. (9) founded the threshold ADC value of 253 lymph nodes in 252 breast cancer patients as 0.986 $\times 10^{-3} \mathrm{~mm}^{2} / \mathrm{sec}$ with sensitivity, specificity, PPV, and NPV values as $75.8 \%, 83.9 \%, 72.6 \%$ and $86.0 \%$, respectively. Fornasa et al. (29) reported the sensitivity and specificity to be $82.22 \%$ and $82.35 \%$, respectively. These results are similar to our percentages that show the reproducibility of ADC measurement in axillary lymph nodes.

We found a significant difference between ipsilateral metastatic lymph nodes and contralateral non-metastatic ones that; ipsilateral metastatic lymph nodes had lower $A D C$ value (mean $\pm S D$ : $0.77 \pm 0.19 \times 10^{-3}$ ) than contralateral non-metastatic group (mean \pm SD: $1.27 \pm 0.21 \times 10^{-3}$ ). Another study conducted by Ramirez et al. (30) found a similar result. The multivariate logistic regression analysis showed that ADC value lower than $0.972 \times 10^{-3}$ and loss of fatty hilum had the strongest associations with axillary metastasis (Table 4). In the study conducted by Guvenc et al. (27) they also found the strongest associations with lower ADC value $\left(<0.985 \times 10^{-3}\right)$ and axillary metastasis.

\section{Study Limitation}

Our study has some limitations. First of all; due to its retrospective design, the study results couldn't be utilized in clinical diagnosis. A prospective study with a larger number of patients would reveal more trustworthy results. Secondly; all the ADC calculations were obtained by two radiologists by consensus MRI readings which resulted in lack of information about interobserver variability. Lastly; we performed institutional standard protocol to obtain MRIs. As a known dilemma, the calculated ADC values are affected by the scanning parameters (TR and TE) and $b$ value used for DWI. Even so, we believe that this study will contribute to literature regarding the high diagnostic value of diffusion-weighted imaging of axilla in breast cancer patients.

\section{Conclusion}

Diffusion-weighted imaging is significantly valuable in the noninvasive diagnosis of metastatic axillary lymph nodes in breast cancer. In terms of conventional MRI parameters, higher values of the short axis, long axis diameters and loss of fatty hilum are significantly correlated with axillary metastasis. Lower ADC value and loss of fatty hilum had 
strongest associations with axillary metastasis. Therefore; DWI should be included in routine MRI protocols in breast cancer staging, since ADC values of the ipsilateral lymph nodes could help to differentiate the metastatic ones in order to prevent unnecessary biopsies.

\section{Authorship Contributions}

Concept: B.D., B.A.U., Design: B.D., Data Collection or Processing: B.D., B.A.U., Analysis or Interpretation: B.D., B.A.U., Literature Search: B.D., Writing: B.D., B.A.U.

Conflict of Interest: No conflict of interest was declared by the authors.

Financial Disclosure: The authors declared that this study received no financial support.

\section{References}

1. Boyle P, Levin B. World cancer report. Lyon, France: International Agency for Research on Cancer; 2008.

2. Özmen V. Breast Cancer in Turkey: Clinical and Histopathological Characteristics (Analysis of 13.240 Patients). J Breast Health 2014;10:98-105.

3. Jatoi I, Hilsenbeck SG, Clark GM, Osborne CK. Significance of axillary lymph node metastasis in primary breast cancer. J Clin Oncol 1999;17:2334-40.

4. Nemoto T, Natarajan N, Bedwani R, Vana J, Murphy GP. Breast cancer in the medial half. Results of 1978 National Survey of the American College of Surgeons. Cancer 1983;51:1333-8.

5. Yamaguchi $\mathrm{K}$, Schacht $\mathrm{D}$, Nakazono T, Irie H, Abe H. Diffusion weighted images of metastatic as compared with nonmetastatic axillary lymph nodes in patients with newly diagnosed breast cancer. J Magn Reson Imaging 2015;42:771-8.

6. Harnan SE, Cooper KL, Meng Y, et al. Magnetic resonance for assessment of axillary lymph node status in early breast cancer: a systematic review and meta-analysis. Eur J Surg Oncol 2011;37:928-36.

7. Partridge SC. Future applications and innovations of clinical breast magnetic resonance imaging. Top Magn Reson Imaging 2008;19:171-6.

8. Luo N, Su D, Jin $G$, et al. Apparent diffusion coefficient ratio between axillary lymph node with primary tumor to detect nodal metastasis in breast cancer patients. J Magn Reson Imaging 2013;38:824-8.

9. Kim EJ, Kim SH, Kang BJ, et al. Diagnostic value of breast MRI for predicting metastatic axillary lymph nodes in breast cancer patients: diffusion-weighted MRI and conventional MRI. Magn Reson Imaging 2014;32:1230-6.

10. De Cataldo C, Bruno F, Palumbo P, et al. Apparent diffusion coefficient magnetic resonance imaging (ADC-MRI) in the axillary breast cancer lymph node metastasis detection: a narrative review. Gland Surg 2020;9:2225-34.

11. Liu $Y$, Luo H, Wang $C$, et al. Diagnostic performance of T2weighted imaging and intravoxel incoherent motion diffusion- weighted MRI for predicting metastatic axillary lymph nodes in T1 and T2 stage breast cancer [published online ahead of print, 2021 Mar 27]. Acta Radiol 2021;2841851211002834.

12. Valente SA, Levine GM, Silverstein MJ, et al. Accuracy of predicting axillary lymph node positivity by physical examination, mammography, ultrasonography, and magnetic resonance imaging. Ann Surg Oncol 2012;19:1825-30.

13. Mussurakis S, Buckley DL, Horsman A. Prediction of axillary lymph node status in invasive breast cancer with dynamic contrast-enhanced MR imaging. Radiology 1997;203:317-21.

14. Macchini M, Ponziani M, lamurri AP, et al. Role of DCE-MR in predicting breast cancer subtypes. Radiol Med 2018;123:75364.

15. Ahn HS, Jang M, Kim SM, La Yun B, Lee SH. Usefulness of preoperative breast magnetic resonance imaging with a dedicated axillary sequence for the detection of axillary lymph node metastasis in patients with early ductal breast cancer. Radiol Med 2019;124:1220-8.

16. Kul S, Cansu A, Alhan E, Dinc H, Gunes G, Reis A. Contribution of diffusion-weighted imaging to dynamic contrast-enhanced MRI in the characterization of breast tumors. AJR Am J Roentgenol 2011;196:210-7.

17. Kuroki-Suzuki S, Kuroki Y, Nasu K, Nawano S, Moriyama N, Okazaki M. Detecting breast cancer with non-contrast MR imaging: combining diffusion-weighted and STIR imaging. Magn Reson Med Sci 2007;6:21-7.

18. Yilmaz R, Bayramoglu Z, Kartal MG, et al. Stromal fibrosis: imaging features with diagnostic contribution of diffusionweighted MRI. Br J Radiol 2018;91:20170706.

19. Chung J, Youk JH, Kim JA, et al. Role of diffusion-weighted MRI: predicting axillary lymph node metastases in breast cancer. Acta Radiol 2014;55:909-16.

20. Dooms GC, Hricak H, Moseley ME, Bottles K, Fisher M, Higgins CB. Characterization of lymphadenopathy by magnetic resonance relaxation times: preliminary results. Radiology 1985;155:691-7.

21. Bedi DG, Krishnamurthy R, Krishnamurthy $S$, et al. Cortical morphologic features of axillary lymph nodes as a predictor of metastasis in breast cancer: in vitro sonographic study. AJR Am J Roentgenol 2008;191:646-52.

22. Yılmaz E, Erok B, Atca AÖ. Measurement of apparent diffusion coefficient in discrimination of benign and malignant axillary lymph nodes. Pol J Radiol 2019;84:e592-7.

23. Atallah D, Moubarak M, Arab W, et al. MRI-based predictive factors of axillary lymph node status in breast cancer. Breast J 2020;26:2177-82.

24. Yoshimura G, Sakurai T, Oura S, et al. Evaluation of Axillary Lymph Node Status in Breast Cancer with MRI. Breast Cancer 1999;6:249-58.

25. Scaranelo AM, Eiada R, Jacks LM, Kulkarni SR, Crystal P. Accuracy of unenhanced MR imaging in the detection of 
axillary lymph node metastasis: study of reproducibility and reliability. Radiology 2012;262:425-34.

26. Baltzer PA, Dietzel M, Burmeister HP, et al. Application of MR mammography beyond local staging: is there a potential to accurately assess axillary lymph nodes? evaluation of an extended protocol in an initial prospective study. AJR Am J Roentgenol 2011;196:W641-7.

27. Guvenc I, Whitman GJ, Liu P, Yalniz C, Ma J, Dogan BE. Diffusion-weighted MR imaging increases diagnostic accuracy of breast MR imaging for predicting axillary metastases in breast cancer patients. Breast J 2019;25:47-55.

28. He N, Xie C, Wei W, et al. A new, preoperative, MRI-based scoring system for diagnosing malignant axillary lymph nodes in women evaluated for breast cancer. Eur J Radiol 2012;81:2602-12.

29. Fornasa F, Nesoti MV, Bovo C, Bonavina MG. Diffusionweighted magnetic resonance imaging in the characterization of axillary lymph nodes in patients with breast cancer. J Magn Reson Imaging 2012;36:858-64.

30. Ramírez-Galván YA, Cardona-Huerta S, Elizondo-Riojas G, Álvarez-Villalobos NA, Campos-Coy MA, Ferrara-Chapa CM. Does axillary lymph node size predict better metastatic involvement than apparent diffusion coefficient (ADC) value in women with newly diagnosed breast cancer? Acta Radiol 2020;61:1494-504. 